\title{
THE PALOMAR OBSERVATORY-EUROPEAN OBSERVATORY PHOTOGRAPHIC ATLAS OF THE NORTHERN SKY
}

\author{
S. CLASS, J. QUEBATTE and G. STRIGL \\ European Southern Observatory \\ Garching \\ Germany
}

\section{Introduction}

In 1990, following an agreement with Caltech (Pasadena), the production of the copies of the POSS II was started at ESO. In total, 894 fields, each in three colours (B, R, I), will be copied on-film and on-glass.

The on-film production consists of $125 \mathrm{~B}$-sets, $110 \mathrm{R}$-sets, and $100 \mathrm{I}$-sets. The on-glass production is 8 B-sets, 8 R-sets and 7 I-sets. This corresponds exactly to the number of orders; new orders are only possible if customers default.

The copying work is performed by a team of three photographers, each of whom is dedicated to a specific task within the project. It is expected to be completed within 10 years.

\section{Present Status}

What concerns the original plates, Caltech reports that $66 \%$ of the J-plates, $74 \%$ of the R-plates and $28 \%$ of the $\mathrm{N}$-plates have now been obtained. Of these, we have now copied $301 \mathrm{~B}$-fields on-film, 160 R-fields on-film and 280 B-fields on-glass.

The distribution of the films is made in consignments of 100 fields which are sent about twice a year. The glass plates are sent once a year (to reduce shipping costs) in 200-plate consignments.

\section{The Intermediate Positive Plate}

After receipt of an original plate from Palomar, the first step is to produce two positive copies on-glass. One will be stored at Caltech, the other is used at ESO. Until now, Kodak Process plates have been used both for intermediate positive and negative glass copies.

In order to transfer a maximum amount of information from the original plates, we aim at a background density (sky level) of $1.85 \mathrm{D}$. (ANSI diffuse density) for the positive and gamma $=1.25$. The reason for slightly increasing the gamma (as compared to the previous surveys) is to improve the detection of very faint objects which are close to the background density of the negatives. We aim at a combined (Positive + Negative) gamma $=1.0$ at sky level, 
with a sky density of 0.45 on the film negatives and 0.55 on the glass negatives.

\section{Negative Copies on Film}

Because of their high background density $(1.85 \mathrm{D})$, the intermediate positives cannot be checked directly under the microscope. We therefore make one film copy of each positive to check its quality in terms of sharpness of the stellar images and the presence of unwanted 'photographic stars or galaxies'. Defocussing can be best detected with 50 times magnification at the microscope. To prevent the risk of introducing photographic flaws during the copying process the entire test-film is 'scanned' under the microscope at 12.5 times magnification.

\subsection{THE FILM PRINTER}

The film printer is a Klimsch-Vakuprinter that we have modified for our special purpose. It is fitted with a quartz-iodine $24 \mathrm{~V} / 150 \mathrm{~W}$ lamp above which an anti-heat filter and a Kodak UV filter No. 18A have been placed. Furthermore, there is a film mask, $15 \mathrm{~cm}$ above the lamp, which corrects the cosine effect (15\% radial intensity fall-off). The plate itself rests, emulsion up, on an O-ring in the specially designed aluminium frame at the top of the printer. The surface of the positive is cleaned with compressed air under grazing light, and, if necessary, an anti-static cloth is used to gently remove dust.

\subsection{PROCESSING}

We use Kodak Aerographic Duplicating film for the copies and process them in a Versamat 17 machine. The advantage of this machine is its large tank capacity which enables excellent fixing and washing while running slowly $(1.93 \mathrm{feet} / \mathrm{min}$.) without slowing down the production rate. Dry-to-dry time is $\mathbf{8}$ minutes. The 36.8 litre tanks are filled with Kodak Duraflo RT developer, Kodak 3000 fixer and water.

\section{The Glass Negatives}

We aim at a sky density of $0.55 \mathrm{D}$. The Process plate has a characteristic curve that rises less steeply than that of the Duplicating film. In order to reach a combined gamma $=1.0$ (Positive + Negative) at background level we have to 'shift' the sky density to $0.55 \mathrm{D}$. The reason for this is once more to improve the detection of possibly. new faint objects by slightly increasing the contrast. The increased minimum density does not appear to inconvenience the users.

\section{THE PRINTER}

Our glass-printer was designed specially to make large-format contact copies within the survey projects. The plates are exposed in a horizontal position with a quartz-iodine lamp $25 \mathrm{~V} / 150 \mathrm{~W}$, which is situated in a shaft five metres below. This ensures an extremely good uniformity of illumination. (See also the article in ESO preprint No. 757 by Quebatte \& Dumoulin). Above the lamp there are an aperture, a shutter (linked with the timer), a Kodak UV filter No. 18A, and a large shutter linked with the vacuum pump (dust protection when the lamp is not on). 


\section{Film Quality Control}

When the first film of a series comes out of the machine it has to pass through a series of checks before the series can actually be started. We aim at D. $0.4510 .01 \mathrm{D}$. Five points of the background are measured with the densitometer and averaged. Focus is checked under the microscope. Depending on the colour, we produce between 100 and 125 film copies of each field. Every twentieth film is taken out of the production and thoroughly checked under the microscope.

\section{Glass Plate Quality Control}

Every single plate is visually inspected on a light-table. Two copies of each series undergo close examination under the microscope to detect possible photographic flaws and unsharpness. The density is measured at five points of the background. 